\title{
Approximate Proximal Point Algorithms for Finding Zeroes of Maximal Monotone Operators in Hilbert Spaces
}

\author{
Yeol Je Cho, ${ }^{1}$ Shin Min Kang, ${ }^{2}$ and Haiyun Zhou $^{3}$ \\ ${ }^{1}$ Department of Mathematics Education and the RINS, Gyeongsang National University, \\ Chinju 660-701, South Korea \\ ${ }^{2}$ Department of Mathematics and the RINS, Gyeongsang National University, \\ Chinju 660-701, South Korea \\ ${ }^{3}$ Department of Mathematics, Shijiazhuang Mechanical Engineering College, \\ Shijiazhuang 050003, China
}

Correspondence should be addressed to Haiyun Zhou, witman66@yahoo.com.cn

Received 1 March 2007; Accepted 27 November 2007

Recommended by H. Bevan Thompson

Let $H$ be a real Hilbert space, $\Omega$ a nonempty closed convex subset of $H$, and $T: \Omega \rightarrow 2^{H}$ a maximal monotone operator with $T^{-1} 0 \neq \varnothing$. Let $P_{\Omega}$ be the metric projection of $H$ onto $\Omega$. Suppose that, for any given $x_{n} \in H, \beta_{n}>0$, and $e_{n} \in H$, there exists $\bar{x}_{n} \in \Omega$ satisfying the following set-valued mapping equation: $x_{n}+e_{n} \in \bar{x}_{n}+\beta_{n} T\left(\bar{x}_{n}\right)$ for all $n \geq 0$, where $\left\{\beta_{n}\right\} \subset(0,+\infty)$ with $\beta_{n} \rightarrow+\infty$ as $n \rightarrow \infty$ and $\left\{e_{n}\right\}$ is regarded as an error sequence such that $\sum_{n=0}^{\infty}\left\|e_{n}\right\|^{2}<+\infty$. Let $\left\{\alpha_{n}\right\} \subset(0,1]$ be a real sequence such that $\alpha_{n} \rightarrow 0$ as $n \rightarrow \infty$ and $\sum_{n=0}^{\infty} \alpha_{n}=\infty$. For any fixed $u \in \Omega$, define a sequence $\left\{x_{n}\right\}$ iteratively as $x_{n+1}=\alpha_{n} u+\left(1-\alpha_{n}\right) P_{\Omega}\left(\bar{x}_{n}-e_{n}\right)$ for all $n \geq 0$. Then $\left\{x_{n}\right\}$ converges strongly to a point $z \in T^{-1} 0$ as $n \rightarrow \infty$, where $z=\lim _{t \rightarrow \infty} J_{t} u$.

Copyright (C) 2008 Yeol Je Cho et al. This is an open access article distributed under the Creative Commons Attribution License, which permits unrestricted use, distribution, and reproduction in any medium, provided the original work is properly cited.

\section{Introduction and preliminaries}

Let $H$ be a real Hilbert space with the inner product $\langle\cdot, \cdot\rangle$ and norm $\|\cdot\|$. A set $T \subset H \times H$ is called a monotone operator on $H$ if $T$ has the following property:

$$
\left\langle x-x^{\prime}, y-y^{\prime}\right\rangle \geq 0, \quad \forall(x, y),\left(x^{\prime}, y^{\prime}\right) \in T .
$$

A monotone operator $T$ on $H$ is said to be maximal monotone if it is not properly contained in any other monotone operator on $H$. Equivalently, a monotone operator $T$ is maximal monotone if $R(I+t T)=H$ for all $t>0$. For a maximal monotone operator $T$, we can define the resolvent 
of $T$ by

$$
J_{t}=(I+t T)^{-1}, \quad \forall t>0 .
$$

It is well known that $J_{t}: H \rightarrow D(T)$ is nonexpansive. Also we can define the Yosida approximation $T_{t}$ by

$$
T_{t}=\frac{1}{t}\left(I-J_{t}\right), \quad \forall t>0
$$

We know that $T_{t} x \in T J_{t} x$ for all $x \in H,\left\|T_{t} x\right\| \leq|T x|$ for all $x \in D(T)$, where $|T x|=\inf \{\|y\|: y \in$ $T x\}$, and $T^{-1} 0=F\left(J_{t}\right)$ for all $t>0$.

Throughout this paper, we assume that $\Omega$ is a nonempty closed convex subset of a real Hilbert space $H$ and $T: \Omega \rightarrow 2^{H}$ is a maximal monotone operator with $T^{-1} 0 \neq \varnothing$.

It is well known that, for any $u \in H$, there exists uniquely $y_{0} \in \Omega$ such that

$$
\left\|u-y_{0}\right\|=\inf \{\|u-y\|: y \in \Omega\} .
$$

When a mapping $P_{\Omega}: H \rightarrow \Omega$ is defined by $P_{\Omega} u=y_{0}$ in (1.4), we call $P_{\Omega}$ the metric projection of $H$ onto $\Omega$. The metric projection $P_{\Omega}$ of $H$ onto $\Omega$ has the following basic properties:

(i) $\left\langle P_{\Omega} x^{\prime}-x, x^{\prime}-P_{\Omega} x^{\prime}\right\rangle \geq 0$ for all $x \in \Omega$ and $x^{\prime} \in H$,

(ii) $\left\|P_{\Omega} x-P_{\Omega} y\right\|^{2} \leq\left\langle x-y, P_{\Omega} x-P_{\Omega} y\right\rangle$ for all $x, y \in H$,

(iii) $\left\|P_{\Omega} x-P_{\Omega} y\right\| \leq\|x-y\|$ for all $x, y \in H$,

(iv) $x_{n} \rightarrow x_{0}$ weakly and $P x_{n} \rightarrow y_{0}$ strongly imply that $P x_{0}=y_{0}$.

Finding zeroes of maximal monotone operators is the central and important topic in nonlinear functional analysis. A classical method to solve the following set-valued equation:

$$
0 \in T z
$$

where $T: \Omega \rightarrow 2^{H}$ is a maximal monotone operator, is the proximal point algorithm which, starting with any point $x_{0} \in H$, updates $x_{n+1}$ iteratively conforming to the following recursion:

$$
x_{n} \in x_{n+1}+\beta_{n} T x_{n+1}, \quad \forall n \geq 0,
$$

where $\left\{\beta_{n}\right\} \subset[\beta, \infty), \beta>0$, is a sequence of real numbers. However, as pointed out in [1], the ideal form of the algorithm is often impractical since, in many cases, solving the problem (1.6) exactly is either impossible or as difficult as the original problem (1.5). Therefore, one of the most interesting and important problems in the theory of maximal monotone operators is to find an efficient iterative algorithm to compute approximately zeroes of $T$.

In 1976, Rockafellar [2] gave an inexact variant of the method

$$
x_{n}+e_{n+1} \in x_{n+1}+\beta_{n} T x_{n+1}, \quad \forall n \geq 0,
$$

where $\left\{e_{n}\right\}$ is regarded as an error sequence. This method is called an inexact proximal point algorithm. It was shown that if $\sum_{n=0}^{\infty}\left\|e_{n}\right\|<+\infty$, then the sequence $\left\{x_{n}\right\}$ defined by (1.7) converges weakly to a zero of $T$. Güler [3] constructed an example showing that Rockafellar's proximal point algorithm (1.7) does not converge strongly, in general. This gives rise to the following question. 
Question 1. How to modify Rockafellar's algorithm so that strong convergence is guaranteed?

$\mathrm{Xu}$ [4] gave one solution to Question 1. However, this requires that the error sequence $\left\{e_{n}\right\}$ is summable, which is too strong. This gives rise to the following question.

Question 2. Is it possible to establish some strong convergence theorems under the weaker assumption on the error sequence $\left\{e_{n}\right\}$ given in (1.7)?

It is our purpose in this paper to give an affirmative answer to Question 2 under a weaker assumption on the error sequence $\left\{e_{n}\right\}$ in Hilbert spaces. For this purpose, we collect some lemmas that will be used in the proof of the main results in the next section.

The first lemma is standard and it can be found in some textbooks on functional analysis.

Lemma 1.1. For all $x, y \in H$ and $\lambda \in[0,1]$,

$$
\|\lambda x+(1-\lambda) y\|^{2}=\lambda\|x\|^{2}+(1-\lambda)\|y\|^{2}-\lambda(1-\lambda)\|x-y\|^{2} .
$$

Lemma 1.2 (see [5, Lemma 1]). For all $u \in H, \lim _{t \rightarrow \infty} J_{t} u$ exists and it is the point of $T^{-1} 0$ nearest to $u$.

Lemma 1.3 (see [1, Lemma 2]). For any given $x_{n} \in H, \beta_{n}>0$, and $e_{n} \in H$, there exists $\bar{x}_{n} \in \Omega$ conforming to the following set-valued mapping equation (in short, SVME):

$$
x_{n}+e_{n} \in \bar{x}_{n}+\beta_{n} T \bar{x}_{n}, \quad \forall n \geq 0 \text {. }
$$

Furthermore, for any $p \in T^{-1} 0$, one has

$$
\begin{gathered}
\left\langle x_{n}-p, x_{n}-\bar{x}_{n}+e_{n}\right\rangle \geq\left\langle x_{n}-\bar{x}_{n}, x_{n}-\bar{x}_{n}+e_{n}\right\rangle, \\
\left\|\bar{x}_{n}-e_{n}-p\right\|^{2} \leq\left\|x_{n}-p\right\|^{2}-\left\|x_{n}-\bar{x}_{n}\right\|^{2}+\left\|e_{n}\right\|^{2} .
\end{gathered}
$$

Lemma 1.4 (see [6, Lemma 1.1]). Let $\left\{a_{n}\right\},\left\{b_{n}\right\}$, and $\left\{c_{n}\right\}$ be three real sequences satisfying

$$
a_{n+1} \leq\left(1-t_{n}\right) a_{n}+b_{n}+c_{n}, \quad \forall n \geq 0
$$

where $\left\{t_{n}\right\} \subset[0,1], \sum_{n=0}^{\infty} t_{n}=\infty, b_{n}=\circ\left(t_{n}\right)$, and $\sum_{n=0}^{\infty} c_{n}<\infty$. Then $a_{n} \rightarrow 0$ as $n \rightarrow \infty$.

\section{The main results}

Now we give our main results in this paper.

Theorem 2.1. Let $H$ be a real Hilbert space, $\Omega$ a nonempty closed convex subset of $H$, and $T: \Omega \rightarrow 2^{H}$ a maximal monotone operator with $T^{-1} 0 \neq \varnothing$. Let $P_{\Omega}$ be the metric projection of $H$ onto $\Omega$. Suppose that, for any given $x_{n} \in H, \beta_{n}>0$, and $e_{n} \in H$, there exists $\bar{x}_{n} \in \Omega$ conforming to the SVME (1.9), where $\left\{\beta_{n}\right\} \subset(0,+\infty)$ with $\beta_{n} \rightarrow+\infty$ as $n \rightarrow \infty$ and $\sum_{n=0}^{\infty}\left\|e_{n}\right\|^{2}<+\infty$. Let $\left\{\alpha_{n}\right\}$ be a real sequence in $[0,1]$ such that

(i) $\alpha_{n} \rightarrow 0$ as $n \rightarrow \infty$,

(ii) $\sum_{n=0}^{\infty} \alpha_{n}=\infty$. 
For any fixed $u \in \Omega$, define the sequence $\left\{x_{n}\right\}$ iteratively as follows:

$$
x_{n+1}=\alpha_{n} u+\left(1-\alpha_{n}\right) P_{\Omega}\left(\bar{x}_{n}-e_{n}\right), \quad \forall n \geq 0 .
$$

Then $\left\{x_{n}\right\}$ converges strongly to a fixed point $z$ of $T$, where $z=\lim _{t \rightarrow \infty} J_{t} u$.

Proof

Claim 1. $\left\{x_{n}\right\}$ is bounded.

Fix $p \in T^{-1} 0$ and set $M=\max \left\{\|u-p\|^{2},\left\|x_{0}-p\right\|^{2}\right\}$. First, we prove that

$$
\left\|x_{n}-p\right\|^{2} \leq M+\sum_{j=0}^{n-1}\left\|e_{j}\right\|^{2}, \quad \forall n \geq 0
$$

When $n=0,(2.2)$ is true. Now, assume that (2.2) holds for some $n \geq 0$. We will prove that (2.2) holds for $n+1$. By using the iterative scheme (2.1) and Lemmas 1.1 and 1.3, we have

$$
\begin{aligned}
& \left\|x_{n+1}-p\right\|^{2} \\
& \quad=\alpha_{n}\|u-p\|^{2}+\left(1-\alpha_{n}\right)\left\|P_{\Omega}\left(\bar{x}_{n}-e_{n}\right)-p\right\|^{2}-\alpha_{n}\left(1-\alpha_{n}\right)\left\|u-P_{\Omega}\left(\bar{x}_{n}-e_{n}\right)\right\|^{2} \\
& \quad \leq \alpha_{n} M+\left(1-\alpha_{n}\right)\left\|\bar{x}_{n}-e_{n}-p\right\|^{2} \leq \alpha_{n} M+\left(1-\alpha_{n}\right)\left\|x_{n}-p\right\|^{2}+\left\|e_{n}\right\|^{2} \\
& \quad \leq \alpha_{n} M+\left(1-\alpha_{n}\right) M+\sum_{j=0}^{n}\left\|e_{j}\right\|^{2}=M+\sum_{j=0}^{n}\left\|e_{j}\right\|^{2} .
\end{aligned}
$$

By induction, we assert that

$$
\left\|x_{n}-p\right\|^{2} \leq M+\sum_{j=0}^{n-1}\left\|e_{j}\right\|^{2}<M+\sum_{j=0}^{\infty}\left\|e_{j}\right\|^{2}<+\infty, \quad \forall n \geq 0 .
$$

This implies that $\left\{x_{n}\right\}$ is bounded and so is $\left\{J_{\beta_{n}} x_{n}\right\}$.

Claim 2. $\varlimsup_{n \rightarrow \infty}\left\langle u-z, x_{n+1}-z\right\rangle \leq 0$, where $z=\lim _{t \rightarrow \infty} J_{t} u$, which is guaranteed by Lemma 1.2.

Noting that $T$ is maximal monotone, $u-J_{t} u=t T_{t} u, T_{t} u \in T J_{t} u, x_{n}-J_{\beta_{n}} x_{n}=\beta_{n} T_{\beta_{n}} x_{n}$, $T_{\beta_{n}} x_{n} \in T J_{\beta_{n}} x_{n}$, and $\beta_{n} \rightarrow+\infty(n \rightarrow \infty)$, we have

$$
\begin{aligned}
\left\langle u-J_{t} u, J_{\beta_{n}} x_{n}-J_{t} u\right\rangle & =-t\left\langle T_{t} u, J_{t} u-J_{\beta_{n}} x_{n}\right\rangle \\
& =-t\left\langle T_{t} u-T_{\beta_{n}} x_{n}, J_{t} u-J_{\beta_{n}} x_{n}\right\rangle-t\left\langle T_{\beta_{n}} x_{n}, J_{t} u-J_{\beta_{n}} x_{n}\right\rangle \\
& \leq-\frac{t}{\beta_{n}}\left\langle x_{n}-J_{\beta_{n}} x_{n}, J_{t} u-J_{\beta_{n}} x_{n}\right\rangle \\
& \longrightarrow 0 \quad(n \longrightarrow \infty), \forall t>0
\end{aligned}
$$

and hence

$$
\varlimsup_{n \rightarrow \infty}\left\langle u-J_{t} u, J_{\beta_{n}} x_{n}-J_{t} u\right\rangle \leq 0 .
$$

Note that $\left\|J_{\beta_{n}}\left(x_{n}+e_{n}\right)-J_{\beta_{n}} x_{n}\right\| \leq\left\|e_{n}\right\| \rightarrow 0$ as $n \rightarrow \infty$, and so it follows from (2.6) that

$$
\varlimsup_{n \rightarrow \infty}\left\langle u-J_{t} u, J_{\beta_{n}}\left(x_{n}+e_{n}\right)-J_{t} u\right\rangle \leq 0
$$


Yeol Je Cho et al.

Note that $\left\|P_{\Omega}\left(\bar{x}_{n}-e_{n}\right)-J_{\beta_{n}}\left(x_{n}+e_{n}\right)\right\| \leq\left\|e_{n}\right\| \rightarrow 0$ as $n \rightarrow \infty$ and so it follows from (2.7) that

$$
\varlimsup_{n \rightarrow \infty}\left\langle u-J_{t} u, P_{\Omega}\left(\bar{x}_{n}-e_{n}\right)-J_{t} u\right\rangle \leq 0
$$

Since $\alpha_{n} \rightarrow 0$ as $n \rightarrow \infty$, from (2.1) we have

$$
x_{n+1}-P_{\Omega}\left(\bar{x}_{n}-e_{n}\right) \longrightarrow 0 \quad(n \longrightarrow \infty)
$$

It follows from (2.8) and (2.9) that

$$
\varlimsup_{n \rightarrow \infty}\left\langle u-J_{t} u, x_{n+1}-J_{t} u\right\rangle \leq 0, \quad \forall t>0,
$$

and so, from $z=\lim _{t \rightarrow \infty} J_{t} u$ and (2.10), we have

$$
\varlimsup_{n \rightarrow \infty}\left\langle u-z, x_{n+1}-z\right\rangle \leq 0 .
$$

Claim 3. $x_{n} \rightarrow z$ as $n \rightarrow \infty$.

Observe that

$$
\left(1-\alpha_{n}\right)\left(P_{\Omega}\left(\bar{x}_{n}-e_{n}\right)-z\right)=\left(x_{n+1}-z\right)-\alpha_{n}(u-z)
$$

and so

$$
\left(1-\alpha_{n}\right)^{2}\left\|P_{\Omega}\left(\bar{x}_{n}-e_{n}\right)-P_{\Omega} z\right\|^{2} \geq\left\|x_{n+1}-z\right\|^{2}-2 \alpha_{n}\left\langle u-z, x_{n+1}-z\right\rangle,
$$

which implies that

$$
\left\|x_{n+1}-z\right\|^{2} \leq\left(1-\alpha_{n}\right)\left\|\bar{x}_{n}-e_{n}-z\right\|^{2}+2 \alpha_{n}\left\langle u-z, x_{n+1}-z\right\rangle .
$$

It follows from Lemma 1.3 and (2.14) that

$$
\begin{aligned}
\left\|x_{n+1}-z\right\|^{2} & \leq\left(1-\alpha_{n}\right)\left\|x_{n}-z\right\|^{2}-\left(1-\alpha_{n}\right)\left\|x_{n}-\bar{x}_{n}\right\|^{2}+\left\|e_{n}\right\|^{2}+2 \alpha_{n}\left\langle u-z, x_{n+1}-z\right\rangle \\
& \leq\left(1-\alpha_{n}\right)\left\|x_{n}-z\right\|^{2}+2 \alpha_{n}\left\langle u-z, x_{n+1}-z\right\rangle+\left\|e_{n}\right\|^{2} .
\end{aligned}
$$

Set $\sigma_{n}=\max \left\{\left\langle u-z, x_{n+1}-z\right\rangle, 0\right\}$. Then $\sigma_{n} \rightarrow 0$ as $n \rightarrow \infty$. Indeed, by the definition of $\sigma_{n}$, we see that $\sigma_{n} \geq 0$ for all $n \geq 0$. On the other hand, by (2.11), we know that for arbitrary $\epsilon>0$, there exists some fixed positive integer $N$ such that $\left\langle u-z, x_{n+1}-z\right\rangle \leq \epsilon$ for all $n \geq N$. This implies that $0 \leq \sigma_{n} \leq \epsilon$ for all $n \geq N$, and the desired conclusion follows. Set $a_{n}=\left\|x_{n}-z\right\|^{2}$, $b_{n}=2 \alpha_{n} \sigma_{n}$, and $c_{n}=\left\|e_{n}\right\|^{2}$. Then (2.15) reduces to

$$
a_{n+1} \leq\left(1-\alpha_{n}\right) a_{n}+b_{n}+c_{n}, \quad \forall n \geq 0,
$$

where $\sum_{n=0}^{\infty} \alpha_{n}=\infty, b_{n}=\circ\left(\alpha_{n}\right)$, and $\sum_{n=0}^{\infty} c_{n}<+\infty$. Thus it follows from Lemma 1.4 that $a_{n} \rightarrow 0$ as $n \rightarrow 0$, that is, $x_{n} \rightarrow z \in T^{-1} 0$ as $n \rightarrow \infty$. This completes the proof. 
Remark 2.2. The maximal monotonicity of $T$ is only used to guarantee the existence of solutions to the SVME (1.9) for any given $x_{n} \in H$ and $\beta_{n}>0$. If we assume that $T: \Omega \rightarrow 2^{H}$ is monotone (need not be maximal) and $T$ satisfies the range condition

$$
\overline{D(T)}=\Omega \subset \bigcap_{r>0} R(I+r T)
$$

then for any given $x_{n} \in \Omega$ and $\beta_{n}>0$, we may find $\bar{x}_{n} \in \Omega$ and $e_{n} \in H$ satisfying the SVME (1.9). Furthermore, Lemma 1.2 also holds for $u \in \Omega$, and hence Theorem 2.1 still holds true for monotone operators which satisfy the range condition.

Following the proof lines of Theorem 2.1, we can prove the following corollary.

Corollary 2.3. Let $H$ be a real Hilbert space, $\Omega$ a nonempty closed convex subset of $H$, and $S: \Omega \rightarrow \Omega$ a continuous and pseudocontractive mapping with a fixed point in $\Omega$. Suppose that, for any given $x_{n} \in \Omega, \beta_{n}>0$, and $e_{n} \in H$, there exists $\bar{x}_{n} \in \Omega$ such that

$$
x_{n}+e_{n}=\left(1+\beta_{n}\right) \bar{x}_{n}-\beta_{n} S \bar{x}_{n}, \quad \forall n \geq 0,
$$

where $\beta_{n} \rightarrow \infty(n \rightarrow \infty)$ and $\left\{e_{n}\right\}$ satisfies the condition $\sum_{n=0}^{\infty}\left\|e_{n}\right\|^{2}<+\infty$. Let $\left\{\alpha_{n}\right\} \subset(0,1]$ be a real sequence such that $\alpha_{n} \rightarrow 0$ as $n \rightarrow \infty$ and $\sum_{n=0}^{\infty} \alpha_{n}=\infty$. For any fixed $u \in \Omega$, define the sequence $\left\{x_{n}\right\}$ iteratively as follows:

$$
x_{n+1}=\alpha_{n} u+\left(1-\alpha_{n}\right) P_{\Omega}\left(\bar{x}_{n}-e_{n}\right), \quad \forall n \geq 0 .
$$

Then $\left\{x_{n}\right\}$ converges strongly to a fixed point $z$ of $S$, where $z=\lim _{t \rightarrow \infty} J_{t} u$, and $J_{t}=(I+t(I-S))^{-1}$ for all $t>0$.

Proof. Let $T=I-S$. Then $T: \Omega \rightarrow 2^{H}$ is continuous and monotone and satisfies the range condition

$$
\overline{D(T)}=\Omega \subset \bigcap_{r>0} R(I+r T) .
$$

Now we only need to verify the last assertion. For any $y \in \Omega$ and $r>0$, define an operator $G: \Omega \rightarrow \Omega$ by

$$
G x=\frac{r}{1+r} S x+\frac{1}{1+r} y .
$$

Then $G: \Omega \rightarrow \Omega$ is continuous and strongly pseudocontractive. By Kamimura et al. [7, Corollary 1], $G$ has a unique fixed point $x$ in $\Omega$, that is, $x=(r /(1+r)) S x+(1 /(1+r)) y$, which implies that $y \in R(I+r T)$ for all $r>0$. In particular, for any given $x_{n} \in \Omega$ and $\beta_{n}>0$, there exist $\bar{x}_{n} \in \Omega$ and $e_{n} \in H$ such that

$$
x_{n}+e_{n}=\bar{x}_{n}+\beta_{n} T \bar{x}_{n}, \quad \forall n \geq 0
$$

which means that

$$
x_{n}+e_{n}=\left(1+\beta_{n}\right) \bar{x}_{n}-\beta_{n} S \bar{x}_{n}, \quad \forall n \geq 0,
$$

and the relation (2.18) follows. The reminder of proof is the same as in the corresponding part of Theorem 2.1. This completes the proof. 
Yeol Je Cho et al.

Remark 2.4. In Corollary 2.3, we do not know wether the continuity assumption on $S$ can be dropped or not.

Remark 2.5. In Theorem 2.1, if the operator $T$ is defined on the whole space $H$, then the metric projection mapping $P_{\Omega}$ is not needed.

Remark 2.6. Our convergence results are different from those results obtained by Kamimura et al. [7].

Theorem 2.7. Let $H$ be a real Hilbert space, $\Omega$ a nonempty closed convex subset of $H$, and $T: \Omega \rightarrow 2^{H}$ a maximal monotone operator with $T^{-1} 0 \neq \varnothing$. Suppose that, for any given $x_{n} \in H, \beta_{n}>0$, and $e_{n} \in H$, there exists $\bar{x}_{n} \in \Omega$ conforming to the following relation:

$$
x_{n}+e_{n} \in \bar{x}_{n}+\beta_{n} T \bar{x}_{n}, \quad \forall n \geq 0,
$$

where $\underline{\lim }_{n \rightarrow \infty} \beta_{n}>0$ and $\sum_{n=0}^{\infty}\left\|e_{n}\right\|^{2}<+\infty$. Let $\left\{\alpha_{n}\right\}$ be a sequence in $[0,1]$ with $\varlimsup_{\lim }{ }_{n \rightarrow \infty} \alpha_{n}<1$ and define the sequence $\left\{x_{n}\right\}$ iteratively as follows:

$$
\begin{aligned}
& x_{0} \in \Omega \\
& x_{n+1}=\alpha_{n} x_{n}+\left(1-\alpha_{n}\right) P_{\Omega}\left(\bar{x}_{n}-e_{n}\right), \quad \forall n \geq 0 .
\end{aligned}
$$

Then $\left\{x_{n}\right\}$ converges weakly to a point $p \in T^{-1} 0$.

Proof

Claim 1. $\left\{x_{n}\right\}$ is bounded.

Since $T^{-1} 0 \neq \varnothing$, we can take some $w \in T^{-1} 0$. By using (2.25) and Lemmas 1.1 and 1.3, we obtain

$$
\begin{aligned}
& \left\|x_{n+1}-w\right\|^{2} \\
& \quad=\alpha_{n}\left\|x_{n}-w\right\|^{2}+\left(1-\alpha_{n}\right)\left\|P_{\Omega}\left(\bar{x}_{n}-e_{n}\right)-w\right\|^{2}-\alpha_{n}\left(1-\alpha_{n}\right)\left\|x_{n}-P_{\Omega}\left(\bar{x}_{n}-e_{n}\right)\right\|^{2} \\
& \quad \leq \alpha_{n}\left\|x_{n}-w\right\|^{2}+\left(1-\alpha_{n}\right)\left\|\bar{x}_{n}-e_{n}-w\right\|^{2} \\
& \quad \leq \alpha_{n}\left\|x_{n}-w\right\|^{2}+\left(1-\alpha_{n}\right)\left\|x_{n}-w\right\|^{2}-\left(1-\alpha_{n}\right)\left\|x_{n}-\bar{x}_{n}\right\|^{2}+\left\|e_{n}\right\|^{2} \\
& \quad=\left\|x_{n}-w\right\|^{2}-\left(1-\alpha_{n}\right)\left\|x_{n}-\bar{x}_{n}\right\|^{2}+\left\|e_{n}\right\|^{2} \\
& \quad \leq\left\|x_{n}-w\right\|^{2}+\left\|e_{n}\right\|^{2}
\end{aligned}
$$

and so (2.26) together with $\sum_{n=0}^{\infty}\left\|e_{n}\right\|^{2}<+\infty$ implies that $\lim _{n \rightarrow \infty}\left\|x_{n}-w\right\|^{2}$ exists. Therefore, $\left\{x_{n}\right\}$ is bounded.

Claim 2. $x_{n}-J_{\beta_{n}} x_{n} \rightarrow 0$ as $n \rightarrow \infty$.

It follows from (2.26) that

$$
\left(1-\alpha_{n}\right)\left\|x_{n}-\bar{x}_{n}\right\|^{2} \leq\left\|x_{n}-w\right\|^{2}-\left\|x_{n+1}-w\right\|^{2}+\left\|e_{n}\right\|^{2}
$$

and so (2.26) together with $\varlimsup_{n \rightarrow \infty} \alpha_{n}<1$ implies that

$$
x_{n}-\bar{x}_{n} \longrightarrow 0 \quad(n \longrightarrow \infty) .
$$


Since $\bar{x}_{n}=J_{\beta_{n}}\left(x_{n}+e_{n}\right)$ and $J_{\beta_{n}}$ is nonexpansive, we have

$$
\left\|x_{n}-J_{\beta_{n}} x_{n}\right\| \leq\left\|x_{n}-\bar{x}_{n}\right\|+\left\|\bar{x}_{n}-J_{\beta_{n}} x_{n}\right\| \leq\left\|x_{n}-\bar{x}_{n}\right\|+\left\|e_{n}\right\| \longrightarrow 0
$$

as $n \rightarrow \infty$ and consequently, $x_{n}-J_{\beta_{n}} x_{n} \rightarrow 0$ as $n \rightarrow \infty$.

Claim 3. $\left\{x_{n}\right\}$ converges weakly to a point $p \in T^{-1} 0$ as $n \rightarrow \infty$.

Set $y_{n}=J_{\beta_{n}} x_{n}$ and let $p \in H$ be a weak subsequential limit of $\left\{x_{n}\right\}$ such that $\left\{x_{n_{j}}\right\}$ converges weakly to a point $p$ as $j \rightarrow \infty$. Thus it follows that $\left\{y_{n_{j}}\right\}$ converges weakly to $p$ as $j \rightarrow \infty$. Observe that

$$
\left\|y_{n}-J_{1} y_{n}\right\|=\left\|\left(I-J_{1}\right) y_{n}\right\|=\left\|T_{1} y_{n}\right\| \leq \inf \left\{\|z\|: z \in T y_{n}\right\}=\left\|T_{\beta_{n}} x_{n}\right\|=\left\|\frac{x_{n}-y_{n}}{\beta_{n}}\right\|
$$

By assumption $\underline{\lim }_{n \rightarrow \infty} \beta_{n}>0$, we have

$$
y_{n}-J_{1} y_{n} \longrightarrow 0 \quad(n \longrightarrow \infty)
$$

Since $J_{1}$ is nonexpansive, by Browder's demiclosedness principle, we assert that $p \in F\left(J_{1}\right)=$ $T^{-1} 0$. Now Opial's condition of $H$ guarantees that $\left\{x_{n}\right\}$ converges weakly to $p \in T^{-1}(0)$ as $n \rightarrow \infty$. This completes the proof.

From Theorem 2.7 and the same proof of Corollary 2.3, we have the following corollary.

Corollary 2.8. Let $H$ be a real Hilbert space, $\Omega$ a nonempty closed convex subset of $H$, and $U: \Omega \rightarrow \Omega$ a continuous and pseudocontractive mapping with a fixed point. Set $T=I-U$. Suppose that, for any given $x_{n} \in \Omega, \beta_{n}>0$, and $e_{n} \in H$, there exists $\bar{x}_{n} \in \Omega$ such that

$$
x_{n}+e_{n}=\left(1+\beta_{n}\right) \bar{x}_{n}-\beta_{n} U \bar{x}_{n}, \quad \forall n \geq 0 .
$$

Define the sequence $\left\{x_{n}\right\}$ iteratively as follows:

$$
\begin{gathered}
x_{0} \in \Omega, \\
x_{n+1}=\alpha_{n} x_{n}+\left(1-\alpha_{n}\right) P_{\Omega}\left(\bar{x}_{n}-e_{n}\right), \quad \forall n \geq 0,
\end{gathered}
$$

where $\left\{\alpha_{n}\right\} \subset[0,1]$ with $\overline{\lim }_{n \rightarrow \infty} \alpha_{n}<1,\left\{\beta_{n}\right\} \subset(0,+\infty)$ with $\underline{\lim }_{n \rightarrow \infty} \beta_{n}>0$, and $\left\{e_{n}\right\} \subset H$ with $\sum_{n=0}^{\infty}\left\|e_{n}\right\|^{2}<+\infty$. Then $\left\{x_{n}\right\}$ converges weakly to a fixed point $p$ of $u$.

\section{Applications}

We can apply Theorems 2.1 and 2.7 to find a minimizer of a convex function $f$. Let $H$ be a real Hilbert space and $f: H \rightarrow(-\infty, \infty]$ a proper convex lower semicontinuous function. Then the subdifferential $\mathrm{f} f$ of $f$ is defined as follows:

$$
\partial f(z)=\left\{v^{*} \in H: f(y) \geq f(z)+\left\langle y-z, v^{*}\right\rangle, y \in H\right\}, \quad \forall z \in H .
$$


Theorem 3.1. Let $H$ be a real Hilbert space and $f: H \rightarrow(-\infty, \infty]$ a proper convex lower semicontinuous function. Suppose that, for any $\bar{x}_{n} \in H, \beta_{n}>0$, and $e_{n} \in H$, there exists $\bar{x}_{n}$ conforming to

$$
x_{n}+e_{n} \in \bar{x}_{n}+\beta_{n} \partial f\left(\bar{x}_{n}\right), \quad \forall n \geq 0,
$$

where $\left\{\beta_{n}\right\}$ is a sequence in $(0, \infty)$ with $\beta_{n} \rightarrow \infty(n \rightarrow \infty)$ and $\sum_{n=0}^{\infty}\left\|e_{n}\right\|^{2}<+\infty$. Let $\left\{\alpha_{n}\right\}$ be a sequence in $[0,1]$ such that $\alpha_{n} \rightarrow 0(n \rightarrow \infty)$ and $\sum_{n=0}^{\infty} \alpha_{n}=\infty$. For any fixed $u \in H$, let $\left\{x_{n}\right\}$ be the sequence generated by

$$
\begin{aligned}
& u, x_{0} \in H, \\
& \bar{x}_{n}=\underset{z \in H}{\arg \min }\left\{f(z)+\frac{1}{2 \beta_{n}}\left\|z-x_{n}-e_{n}\right\|^{2}\right\}, \\
& x_{n+1}=\alpha_{n} u+\left(1-\alpha_{n}\right)\left(\bar{x}_{n}-e_{n}\right), \quad \forall n \geq 0 .
\end{aligned}
$$

If $\partial f^{-1} 0 \neq \varnothing$, then $\left\{x_{n}\right\}$ converges strongly to the minimizer of $f$ nearest to $u$.

Proof. Since $f: H \rightarrow(-\infty, \infty]$ is a proper convex lower semicontinuous function, by [2], the subdifferential $\partial f$ of $f$ is a maximal monotone operator. Noting that

$$
\bar{x}_{n}=\underset{z \in H}{\arg \min }\left\{f(z)+\frac{1}{2 \beta_{n}}\left\|z-x_{n}-e_{n}\right\|^{2}\right\}
$$

is equivalent to

$$
0 \in \partial f\left(\bar{x}_{n}\right)+\frac{1}{\beta_{n}}\left(\bar{x}_{n}-x_{n}-e_{n}\right)
$$

we have

$$
x_{n}+e_{n} \in \bar{x}_{n}+\beta_{n} \partial f\left(\bar{x}_{n}\right), \quad \forall n \geq 0 .
$$

Therefore, using Theorem 2.1, we have the desired conclusion. This completes the proof.

Theorem 3.2. Let $H$ be a real Hilbert space and $f: H \rightarrow(-\infty, \infty]$ a proper convex lower semicontinuous function. Suppose that, for any given $x_{n} \in H, \beta_{n}>0$, and $e_{n} \in H$, there exists $\bar{x}_{n} \in H$ such that

$$
x_{n}+e_{n} \in \bar{x}_{n}+\beta_{n} \partial f\left(\bar{x}_{n}\right), \quad \forall n \geq 0,
$$

where $\left\{\beta_{n}\right\}$ is a sequence in $(0, \infty)$ with $\underline{\lim }_{n \rightarrow \infty} \beta_{n}>0$ and $\sum_{n=0}^{\infty}\left\|e_{n}\right\|^{2}<+\infty$. Let $\left\{\alpha_{n}\right\}$ be a sequence in $[0,1]$ with $\varlimsup_{n \rightarrow \infty} \alpha_{n}<1$ and let $\left\{x_{n}\right\}$ be the sequence generated by

$$
\begin{aligned}
& x_{0} \in H, \\
& \bar{x}_{n}=\underset{z \in H}{\arg \min }\left\{f(z)+\frac{1}{2 \beta_{n}}\left\|z-x_{n}-e_{n}\right\|^{2}\right\}, \\
& x_{n+1}=\alpha_{n} x_{n}+\left(1-\alpha_{n}\right)\left(\bar{x}_{n}-e_{n}\right), \quad \forall n \geq 0 .
\end{aligned}
$$

If $\partial f^{-1} 0 \neq \varnothing$, then $\left\{x_{n}\right\}$ converges weakly to the minimizer of $f$ nearest to $u$. 
Proof. As shown in the proof lines of Theorem 3.1, $\partial f: H \rightarrow H$ is a maximal monotone operator, and so the conclusion of Theorem 3.2 follows from Theorem 2.7.

\section{Acknowledgment}

The authors are grateful to the anonymous referee for his helpful comments which improved the presentation of this paper.

\section{References}

[1] J. Eckstein, "Approximate iterations in Bregman-function-based proximal algorithms," Mathematical Programming, vol. 83, no. 1, pp. 113-123, 1998.

[2] R. T. Rockafellar, "Monotone operators and the proximal point algorithm," SIAM Journal on Control and Optimization, vol. 14, no. 5, pp. 877-898, 1976.

[3] O. Güler, "On the convergence of the proximal point algorithm for convex minimization," SIAM Journal on Control and Optimization, vol. 29, no. 2, pp. 403-419, 1991.

[4] H.-K. Xu, "Iterative algorithms for nonlinear operators," Journal of the London Mathematical Society, vol. 66, no. 1, pp. 240-256, 2002.

[5] R. E. Bruck Jr., "A strongly convergent iterative solution of $0 \in U x$ for a maximal monotone operator $U$ in Hilbert space," Journal of Mathematical Analysis and Applications, vol. 48, no. 1, pp. 114-126, 1974.

[6] L. S. Liu, "Ishikawa and Mann iterative process with errors for nonlinear strongly accretive mappings in Banach spaces," Journal of Mathematical Analysis and Applications, vol. 194, no. 1, pp. 114-125, 1995.

[7] S. Kamimura, S. H. Khan, and W. Takahashi, "Iterative schemes for approximating solutions of relations involving accretive operators in Banach spaces," in Fixed Point Theory and Applications, Vol. 5, Y. J. Cho, J. K. Kim, and S. M. Kang, Eds., pp. 41-52, Nova Science Publishers, Hauppauge, NY, USA, 2004. 